\title{
Intron and Its Splicing Mechanism and Their Connection with Human Disease Dosay-Akbulut $M$
}

\begin{abstract}
:
In the maturation mechanism of a messenger RNA, splicing play an important role with removing the noncoding introns and ligating the coding exons. Alternative splicing (AS) gives an extra difficulty to this mechanism and to the regulation of gene expression. The possible disturbing in the alternative RNA splicing mechanism can be a reason to several diseases like cancers and neurodegenerative disorders. Intronless genes (IGs) are seen in almost 3\% of the human genome. Functionality of IGs has an important role in signal transduction genes and related regulatory proteins. This diversity can be reason to IG-associated diseases, especially neuropathies, developmental disorders, and cancer. The retroelements can be seen in almost half of the human genome. The known informations indicate that insertion of retroelement into exons and introns of genes promote different types of genetic disease, including cancer. The retroelement connected mutagenesis cause to fifty different types of human disease. The molecular informations and bioinformatic analyses can be used to explain the connection with splicing mutations and genetic mechanisms of several different human disease and understanding of this mechanism play an important role in the formation of treatment programme against to these diseases.
\end{abstract}

Key Words: splicing; intron; exon; human; disease

Bangladesh Journal of Medical Science Vol. 15 No. 03 July'16. Page : 307-312

\section{Introduction}

Splicing of precursor messenger RNA (pre-mRNA) includes the removing of the intervening sequences (introns) and joining the expressed regions (exons) in the nucleus, before eukaryotic mRNA, containing intron, transcript can be exported and translated into proteins in the cytoplasm. In the constitutive splicing mechanism; some sequences are always included or excluded, but others can be used alternative splicing process. Especially; in the alternative splicing, several thousands of variant transcripts can be produced from a single gene. Disruption of this process create aberrant splicing, with including sevaral defects of up to $50 \%$ of mutations that cause certain human genetic diseases like neurological diseases ${ }^{1}$.

The correct gene splicing is based on the exact recognition of exons. The elements of this process are the "cis" (conserved sequences) and "trans" (snRNP, splicing factors) elements. Splicing mutations are reason of several different genetic disorders and usually cause to exon skipping, activate new cryptic exons or form new exon/ intron boundaries as a result of alterations at donor/acceptor sites. They form more than $9 \%$ of the known mutations, but this value is generally ignored because of many potential mutations located in the "cis" elements and these should be confirmed experimentally. The most known splicing mutations are located at donor (5') and acceptor (3') sites; in contrast to rarely seen mutations of polypyrimidine tract and at the branch point of splicing mechanism. High throughput technologies, and Sanger sequencing, allow detection of many changes in intron/exon boundaries and in intronic sequences. But the detection of mutation affects onto genetic disorder especially for diagnostic purposes can be managed by using molecular biology methods, such as; in vitro transcription of the sequence of interest cloned into a plasmid, with and without alterations, or mutation analysis via a hybrid minigene system ${ }^{2}$. The precursor transcript of eukaryotic gene (premRNA) is composed of coding regions--exons inserted by non-coding introns, which are removed in the RNA splicing process, finalized to the formation of mature, intron-free mRNA. Alternative splicing of pre-mRNA is based on the high complexity of the cellular proteome and expresses effective use of genetic information contained in genomic DNA. Alternative splicing has an important role

Corresponds to: Mine Dosay-Akbulut, Medical Biology and Genetics Department, Veterinary Faculty, Afyon Kocatepe University, AFYON-TURKEY, E-mail: minedosay@aku.edu.tr 
in the organism, such as; apoptosis regulation or development and plasticity of the nervous system. Because of the high complexity of this mechanism, alternative splicing is particularly inclined to errors. The error, caused from mutations, in the key sequences for splicing regulations are especially important. Hence the disturbed alternative RNA splicing can cause to several diseases such as cancers and neurodegenerative disorders. The treatment and succeed of these conditions have a trouble because of their genetic background and currently RNA interference. The effort of silencing the expression of incorrect protein isoforms support the idea of using RNA interference in aimed disease related to problem in alternative splicing of RNA ${ }^{3}$. Intronless genes (IGs) are found in almost $3 \%$ of the human genome. Human IGs are different from the IGs of unicellular eukaryotes on the basis of functionality and in evolution. Functionality of IGs determined an important role of signal transduction genes and related regulatory proteins, on growth, proliferation, and development. These differences caused to IG-associated diseases, especially neuropathies, developmental disorders, and cancer ${ }^{4}$. In a transcription mechanism as a part of gene expression of most mammalian genes, the removal of introns via splicing is an important step. The splicing mechanism includes complex ribonucleoprotein particles named as spliceosomes. There are two types of introns that splice out mutually in mammals. One of them is minor spliceosome, is linked to a rare autosomal recessive developmental defect with its mutations in snRNA, U4atac. On the other side; it was seen somatic, heterozygous mutations related to other splicing factors causing to particular cancers and pre-cancerous conditions at high frequencies, suggesting the core splicing machinery defects has an important role in tumorigenesis. The identification of these genes and understanding the mis-splicing mechanisms provide an advantage in diagnosis and treatment ${ }^{5}$. The splicing has a role on maturation mechanism of a messenger RNA. Alternative splicing (AS) adds an extra complexity to this mechanism and to the regulation of gene expression. This extra splicing cause multiple messenger RNA isoforms from a single gene with creating different exon-intron inclusions and exclusions and alternative splice sites. With the effect of AS, it can be seen the increasity in the coding potential of eukaryotic genomes and the diversity of eukaryotic proteomes. The result of mutations, via abolish of either constitutive splicing or AS, several diseases such as; myotonic dystrophy and cystic fibrosis can be formed. Especially the specific splicing is determined clearly in cancer states. The identification of rare orijinal mutations, related to splice-site recognition, and splicing regulation, give an advantage to understand genetic mechanisms of rare diseases. Also; the molecular exome sequencing and bioinformatic analyses provide informations to explain the connection with splicing mutations and human disease ${ }^{6}$.

There are also retroelements within noncoding DNAs; which placed almost half of the human genome. L1, Alu and SVA are example to them. They pass via retrotransposition and migrates to genomic regions with 5' and 3' transduction, as a result promote or inhibit gene transcription via using alternative promoters or constituting antisense regulatory noncoding RNAs. There is effection of Retroelements into the expression of genes with exonization using cryptic splice sites, by providing polyadenylation signals and alternative splicing via exon skipping. The recent findings indicate that insertion of retroelement into exons and introns of genes induce different types of genetic disease, including cancer. The retroelement linked mutagenesis, lead to fifty different types of human disease ${ }^{7}$.

As a result of AS procedure and related mutations several different disease can be seen such as;

\section{Sub dividing}

\section{Tailoring}

Alternative splicing (AS) of RNA has an important role on diversification of the eukaryotic proteome. In this process, different mRNA transcripts can be obtained via altered excision and/or inclusion of exons during splicing of the pre-mRNA molecule. It was determined that AS has a significant role in protein structure, function, and localization. A possible defect in this process can result with disease phenotypes. Also, it is believed that AS pathways are key factor as therapeutic targets for a number of diseases such as Alzheimer's disease. Integral membrane proteins (MPs) are class of proteins that specifically adaptable to regulation via alternative splicing because of their topological restriction related to their folding, structure, mechanism and function ${ }^{8}$.

\section{Neurologic Disease}

Alternative pre-mRNA splicing effect the protein outcome of most neuronally expressed genes. The coding changes of different mRNA isoforms can adjust the protein function of that. It was showed 
that alternative splicing can play a role as an on/ off switch for gene expression. Also some other regulated splicing events, may affect the benefit outcome of mRNA in its later cytoplasmic life via changing its localization or translation. It was clearly understand that the multiple steps of post-transcriptional gene regulation are strongly connected. All of these regulatory process, play key roles in all stages of the cell biology of neurons, from their initial differentiation, to function with mature circuits ${ }^{9}$.

\section{Hematological disorders}

In humans, the vast of all protein-coding transcripts include introns that are removed by mRNA splicing with the help of spliceosomes. Within the spliceosome machinery, some mutations have been determined with using whole-exome/ genome technologies in myelodysplastic syndromes (MDS) and in other hematological disorders. As a spliceosomal mutations, firstly the changings in splicing factor 3 subunit b1 (SF3b1) were obtained. After that, some other splicing factor mutations such as U2 small nuclear RNA auxillary factor 1 (U2AF1) and serine arginine-rich splicing factor 2 (SRSF2). SF3b1/U2AF1/SRSF2 mutations can be seen at varying frequencies in different disease subtypes, especially for MDS and other hematological malignancies each affecting the differences in survival outcomes ${ }^{10}$.

\section{Liver disease}

Pre-mRNA splicing is an important step in gene expression of eukaryotes with including removal of introns and connection of exons to obtain mature mRNAs. This is a highly regulated mechanism that includes the alternative usage of exons, the retention of intronic sequences and the creation of exonic sequences with different length. The splicing events cab be seen in most human genes and disruptions of this process have been related with different kind of diseases, including cancer. Hepatocellular carcinoma (HCC) is a kind of tumor that usually develops in a cirrhotic liver. The changings in premRNA splicing of some genes have been showed in liver cancer, and the obtained informations indicate that splicing defects may have a role in hepatocarcinogenesis ${ }^{11}$.

\section{Metabolic Disease}

The several number of mutations determined in introns which activate or create novel splice sites resulting the fake exon inclusion in mRNA causing to inherited metabolic disease (IMD) and other human genetic diseases. The specific feature in that the native splice sites remain intact with a potential for normal splicing.

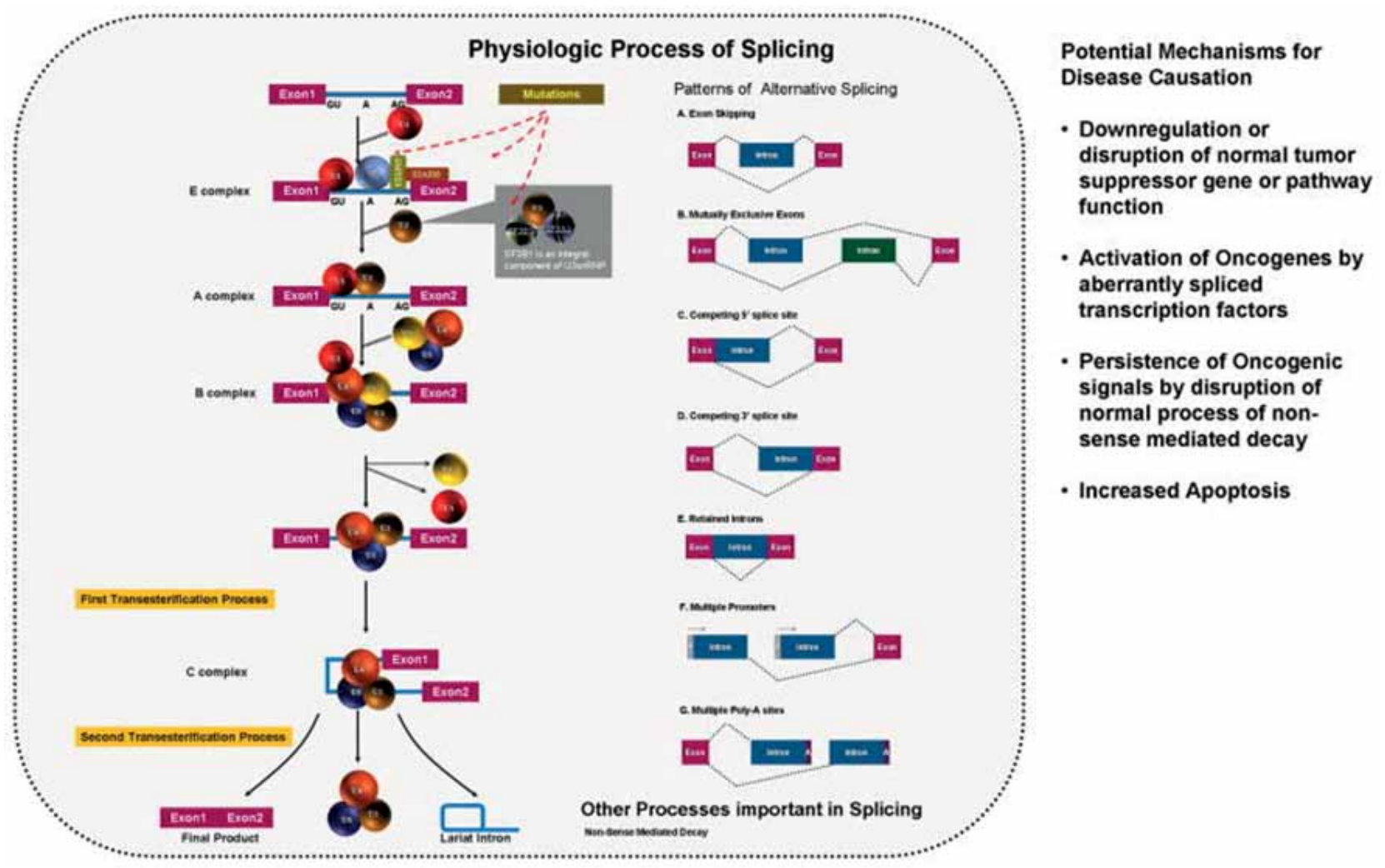

Fig.1: Alternative splicing pattern and disease causation [10]. 
It was determined that antisense oligonucleotides (AO) adjust the splicing pattern by steric hindrance of the recognition and binding of the splicing facilities to the given sequences. In the pseudoexon events, AO give pressure to use of the natural splice sites, regaining normally spliced transcripts resulted with functional protein. Some positive results were obtained from antisense splicing modulation as a molecular therapy approach for pseudoexon-activating mutations, resulted with IMD disease and Duchenne muscular dystrophy ${ }^{12}$.

\section{Therapeutic strategies}

It was understood that the aberrant pseudoexon inclusion as a reason of human disease is more frequent than previously thought on the basis of screening studies ${ }^{13}$.

In higher eukaryotes, the protein-coding genes are transcribed as pre-mRNA which means the coding regions (exons) are separated by noncoding segments (introns). The process of exons connection, to give the mature mRNA, is called splicing. Almost $90 \%$ of all human genes are alternatively spliced, indicating alternative splicing affect positively proteome diversity and important regulatory functions. It was determined that mutations related to splicing regulation have an affect in the formation of several diseases. The repair of these disturbed splicing processes can be used as a possible therapeutic concept and in the development of several drugs ${ }^{14}$.

Different bacterial fermentation products and their synthetic coproducts show antitumour activities and connect to components of the spliceosome, which plays an important role in the splicing mechanism of eukaryotic cells. The changings in the gene expression, including alternative splicing, are important for cancer progression. The recent reports showed that the splicing factors coding genes, including the drug target splicing factor $3 \mathrm{~B}$ subunit 1 (SF3B1), are among the utmost mutated in several haematological malignancies such as chronic lymphocytic leukaemia and myelodysplastic syndromes. These findings reveal the role of splicing factors and the spliceosome in cancer and suggest the use of drugs, targeting these proteins as novel antitumour therapies ${ }^{15}$.

\section{Heart Disease}

Different protein products, from each gene are possible with using alternative splicing. The differences in exon connection, intron removing or the use of alternative splice sites can change protein structure, localisation, regulation and function.
In the heart, alternative splicing of ion channels, cell signalling proteins and sarcomeric genes, can cause to cardiomyopathies, arrhythmias and other pathologies. In addition, the altered splicing, because of mutations can lead to several inherited conditions and heart-related diseases also ${ }^{16}$.

\section{Some other Disease}

Epigenetics is a process that has an effect on gene expression programs without changes in DNA sequence. The main factors in epigenetic control are chemical modifications to DNA, histone, and non-histone chromosomal proteins, they together act as a regulatory network to control genome function. The methylation of DNA by DNA methyltransferases at the fifth position of cytosine in $\mathrm{CpG}$ dinucleotides (5-methylcytosine, $5 \mathrm{mC}$ ), is associated with gene silencing. But deteailed study of DNA methylation indicated that $5 \mathrm{mC}$ is full of with exonic nucleosomes and intron-exon junctions, suggesting DNA methylation has a role in the relationship between elongation and RNA splicing.

The changings in DNA methylation models are known to be related with several human diseases. The main mechanism in this changing leans to participation of MBPs (methyl binding proteins). MBPs can cause human diseases via different ways. These ways are based on alterations in (1) transcriptional adjustment (transcription, splicing, posttranscriptional regulation); (2) protein expression and dealing with different functional cellular compartments; (3) expression in specific cell types; (4) determination of position (nuclear, cytoplasmic, nucleoli); (5) mutations lead to damaged expression, localization or functions or binding to interacting proteins/DNA; (6) posttranslational modifications as a reason of damaged function; and (7) binding to chromatin and target genes. The existence of these structures are related with several human diseases such as cancer, diabetes, imprinting disorders, and immune system-related disorders.

Cancer was accepted as a genetic disease caused by mutations, but recent informations indicated that alterations in the epigenome also have a role. Correct methylation occurrence is necessary for the suitable binding of MBPs. Different roles of MBPs can be seen in different types of cancers ${ }^{17}$.

Alternative splicing and their role in cancer is also getting more interest, as both changes in splicing factor expression and cis-mutations can cause disease. Mutations in cisacting motifs, usually 
the reason of different changes in splicing and this mechanism can be seen in oncogenes such as BRCA1 (breastcancer susceptibility gene 1) ${ }^{18}$.

Diabetes is one of the most important metabolic disorders. The reason to this disorder either lack of insulin or an apathy to insulin, which cause to high blood glucose levels and chronic damage in several organ systems. Among the effected factors of diabetes, epigenetic modifications have taken interest because they can put a link between its pathology and environmental cues. The methioninehomocysteine cycle, is very important in DNA methylation. The changings in DNA methylation caused by CpG-SNPs are also common in diabetes. Because different diabetes-related genes are controlled by DNA methylation, it is most probably other MBPs have a role on the pathogenesis of diabetes or related metabolic disorders.

\section{Imprinted Disorders}

Imprinting disorders like Prader-Willi syndrome (PWS)/Angelman syndrome (AS), BeckwithWeidemann syndrome, and Silver-Russell syndrome are related with a mutation or deletion in part of DMRs (differentially methylated regions). Alterations in MBPs may be associated with dysregulation of imprinted genes.

\section{Immune System-Related Disorders}

Hematopoietic stem cell (HSCs) differentiation needs firmly controlled gene expression form, which comes from epigenetic regulation and many of the hematopoiesis-related genes are controlled by promoter methylation. Changings in expression and mutations of DNMTs (DNA methyltransferases) have been linked to impaired hematopoiesis, which cause to immune disorders; including allergies and autoimmune disease.

\section{Cardiovascular Diseases and Cerebral Ischemia}

The effect of epigenetic factors on cardiovascular disease was based on DNA methylation and microRNAs. The global DNA hypomethylation and increased S-adenosylhomocysteine pattern have been determined in vascular disease. It was determined that DNA methylation effect several genes by misregulating, which could lead to cardiovascular diseases.

Also; loss of function of DNA methyltransferases cause to increased resistance to brain damaged by ischemia and together, it was shown that; there is a possible link between DNA methylation and cerebral ischemia ${ }^{17}$.

\section{Conclusions}

The pre-mRNA of eukaryotic gene is formed by coding regions--exons and non-coding introns-which are removed in the RNA splicing process, finalized to the formation of mature, intron-free mRNA.

The correct gene splicing is based on the exact recognition of exons. The possible mistake in the splicing mechanism via mutations or alternative splicing, are reason of several different genetic disorders and usually lead to exon skipping, activate new cryptic exons or form new exon/ intron boundaries as a result of changings at donor/acceptor sites. With the effect of AS, it was determined that there is an increasity in the coding potential of eukaryotic genomes and the diversity of eukaryotic proteomes.

As a consequence of mutations, cause to damage in splicing mechanism or to AS, several diseases such as; myotonic dystrophy and cystic fibrosis can be seen. Especially the effect of changings in splicing has been determined in cancer states. The determination of this kind of mutations, helps to understand genetic mechanisms of rare diseases. These informations with the help of molecular exome sequencing and bioinformatic datas, give an advantage to explain the connection with splicing mutations and human disease and provide an assist to find effective treatment against to these illness and to form health plan in general.

Conflict of interest: None declared 


\section{Referances:}

1. Feng, D.; Xie, J. Aberrant splicing in neurological diseases. Wiley Interdiscip. Rev. RNA. 2013, 4(6), 631-49.

2. Lewandowska, M.A. The missing puzzle piece: splicing mutations. Int. J. Clin. Exp. Pathol. 2013, 6(12), 2675-82.

3. Wysokiński, D.; Błasiak, J. Perspectives of RNA interference application in the therapy of diseases associated with defects in alternative RNA splicing. Postepy. Hig. Med. Dosw. 2012, 66, 683-95.

4. Grzybowska, E.A. Human intronless genes: functional groups, associated diseases, evolution, and mRNA processing in absence of splicing. Biochem. Biophys. Res. Commun. 2012, 424(1), 1-6.

5. Padgett, R.A. New connections between splicing and human disease. Trends. Genet. 2012, 28(4), 147-54.

6. Taneri, B.; Asilmaz, E.; Gaasterland, T. Biomedical impact of splicing mutations revealed through exome sequencing. Mol. Med. 2012, 18, 314-9.

7. Kaer, K.; Speek, M. Retroelements in human disease. Gene, 2013, 518(2), 231-41.

8. Mittendorf, K.F.; Deatherage, C.L.; Ohi, M.D.; Sanders, C.R. Tailoring of membrane proteins by alternative splicing of pre-mRNA. Biochemistry. 2012, 51(28), 5541-56.

9. Zheng, S.; Black, D.L. Alternative pre-mRNA splicing in neurons: growing up and extending its reach. Trends Genet. 2013, 29(8), 442-8.

10. Visconte, V.; Makishima, H.; Maciejewski, J.P.; Tiu, R.V. Emerging roles of the spliceosomal machinery in myelodysplastic syndromes and other hematological disorders. Leukemia. 2012, 26(12), 2447-54.
11. Berasain, C.; Goñi, S.; Castillo, J.; Latasa, M.U.; Prieto, J.; Avila, M.A. Impairment of pre-mRNA splicing in liver disease: mechanisms and consequences. World J. Gastroenterol. 2010, 16(25), 3091-102.

12. Pérez, B.; Rodríguez-Pascau, L.; Vilageliu, L.; Grinberg, D.; Ugarte, M.; Desviat, L.R. Present and future of antisense therapy for splicing modulation in inherited metabolic disease. J. Inherit. Metab. Dis. 2010, 33(4), 397-403.

13. Dhir, A.; Buratti, E. Alternative splicing: role of pseudoexons in human disease and potential therapeutic strategies. FEBS J. 2010, 277(4), 841-55.

14. Heyd, F. Alternative splicing--principles, functional consequences and therapeutic implications. Dtsch. Med. Wochenschr. 2014, 139(7), 339-42.

15. Bonnal, S.; Vigevani, L.; Valcárcel, J. The spliceosome as a target of novel antitumour drugs. Nat. Rev. Drug. Discov. 2012, 11(11), 847-59.

16. Lara-Pezzi, E.; Gómez-Salinero, J.; Gatto, A.; GarcíaPavía, P. The alternative heart: impact of alternative splicing in heart disease. J. Cardiovasc. Transl. Res. 2013, 6(6), 945-55.

17. Liyanage, V.R.; Jarmasz, J.S.; Murugeshan, N.; Del Bigio, M.R.; Rastegar, M.; Davie, J.R. DNA modifications: function and applications in normal and disease States. Biology. (Basel). 2014, 3(4), 670-723.

18. Jensen, C.J.; Oldfield, B.J.; Rubio, J.P. Splicing, cis genetic variation and disease. Biochem. Soc. Trans. 2009, 37(Pt 6), 1311-5. 